Invited Papers

\title{
Near-infrared absorption detection in picolitre liquid volumes using amplified fibre loop ring-down detection
}

\author{
Nicholas L.P. Andrews, Jessica Litman, Daniel Stroh, Jack A. Barnes, Hans-Peter Loock* \\ Dept. of Chemistry, Queen's University, Kingston, ON K7L 3N6, Canada
}

\section{A R T I C L E I N F O}

Article history:

Available online 29 August 2013

\section{Keywords}

Cavity ring-down

Absorption spectroscopy

Fiber (fibre) loop

Acetylene gas detection

Liquid sensing

Picoliter (picolitre) volume

\begin{abstract}
A B S T R A C T
Cavity ring-down spectroscopy is performed using a single-mode fibre loop as an optical cavity. The loop contains absorption cells for either liquids or gases, as well as an erbium-doped fibre amplifier. The fibre amplifier is gain-clamped using a second fibre-loop. Ring-down times can be tuned to permit several hundred roundtrips by adjusting the loss ratio between the two nested loops using a variable optical attenuator. Ring-down measurements were performed either in the time domain or using phase shift measurements.

Acetylene gas was detected through a ro-vibrational absorption line of the $v_{1}-v_{3}$ combination band at $1532 \mathrm{~nm}$ at a limit of detection of $50 \mathrm{ppm}$. Liquid 1-octyne and 1-dodecyne were detected in a $1 \mathrm{pL}$ volume at detection limits of $\sim 20 \%$ dilution in dodecane through their $\mathrm{C}-\mathrm{H}$ stretch vibrational overtone transition.
\end{abstract}

(c) 2013 Elsevier Inc. All rights reserved.

\section{Introduction}

Over recent years cavity ring-down spectroscopy (CRDS) and related cavity-enhanced absorption techniques have gained a large following. In CRDS light is coupled into a high finesse optical cavity and one measures the optical loss from the rate at which light escapes from the cavity. The decay time or "ring-down time", $\tau$, represents the lifetime of a photon trapped within a cavity and is a direct measure of the optical loss in the cavity [1]. Importantly the ring-down time is immune to intensity variations [2]. Adding an absorbing sample into the cavity creates additional losses and the consequent decrease of the ring-down time allows for an accurate determination of the concentration of the sample [3].

Most CRD spectrometers are mirror-based and are designed for sensitive trace gas [4] or aerosol [5,6] absorption measurements with effective optical path lengths of several $\mathrm{km}$ to enhance detection [7]. Measurements on liquids require either filling the entire cavity with liquid or placing the sample into a part of the cavity-for example in a cuvette [8]. Using liquids in a "conventional" cavity is complicated by the requirement to minimise losses due to the scattering of light at the interfaces of the liquid and sample containers, but excellent detection limits have been achieved in a variety of configurations [9].

An alternative approach is to construct the optical cavity from optical fibres rather than mirrors [10-12]. Here, the ring-down time is calculated from the roundtrip transmission of the empty

\footnotetext{
* Corresponding author.

E-mail address: hploock@chem.queensu.ca (H.-P. Loock).
}

cavity, $T_{\text {sys, }}$, the transmission through the sample, $T_{\text {sample, }}$ and the roundtrip time $t_{r t}=n L / c_{0}$ where $n$ is the refractive index of the optical fibre, $L$ is the length of the loop and $c_{0}$ is the speed of light in vacuum.

$$
\tau=-\frac{t_{r t}}{\ln \left(T_{\text {sys }}\right)+\ln \left(T_{\text {sample }}\right)}
$$

On one hand round-trip losses, $L_{\text {sys }}[\mathrm{dB}]=-4.34 \ln \left(T_{\text {sys }}\right)$, tend to be much higher for such cavities, due to optical losses in fibre splices, couplers, and sample interfaces as well as the fibre material itself. On the other hand the setup can be made very robust and inexpensive and can be readily interfaced to microanalytical separation systems such as capillary electrophoresis [13], or microfluidic devices [14]. Light can be coupled into the loop in a variety of different ways using either commercial fibre-fibre couplers [15], custom interfaces [16], small notches in the fibre cladding [17], or simply a bend in the fibre [10]. The main advantage of fibre-CRD spectroscopy over mirror based setups is that it only requires sample volumes of $\mathrm{nL}$ or even $\mathrm{pL}[18,19]$ rather than volumes of several $\mu \mathrm{L}$ [20-24]. Furthermore, fibre CRD spectrometers are readily aligned and comparably compact. Recent reviews describe a variety of different implementations of this concept as well as their application to different (micro-) analytical problems [1,25].

For fibre-CRD spectroscopy there are generally two methods of introducing the absorbing sample. One can either introduce the sample within a cleave of the fibre [10], or one can expose the sample solution to the evanescent wave of the core mode $[12,26]$. The latter can be achieved using field access blocks [27], tapers [26], or by removing the cladding of the fibre optic cable [12]. 
Finally, fibre-CRD spectroscopy can be implemented with either a linear cavity or in a loop [28]. Loop cavities are preferred when a very large spectral bandwidth is required. Here the bandwidth is only limited by the optical transmission of the fibre loop and its components and can be as large as $300 \mathrm{~nm}$ to $1650 \mathrm{~nm}$. This is of advantage if multiple analytes have to be detected simultaneously in a wide wavelength range [29]. Linear cavities require fibre Bragg gratings or dielectric coatings that have comparably narrow reflection bands, and are more difficult to fabricate. However, loop cavities do have the problem of far larger system losses, $L_{\text {sys }}$ caused by the implementation of couplers, splices and absorption cells which may add up to a total loss that is larger than the loss introduced by the absorption of the sample, $L_{\text {sample. }}$.

Already in 2001, Stewart et al. proposed and implemented a conceptually simple method to compensate for the losses in a fibre loop cavity by introducing a gain element, $G$, into the loop [30,31]. The ring-down time then becomes

$$
\tau=-\frac{t_{r t}}{\ln \left(T_{\text {sys }}\right)+\ln \left(T_{\text {sample }}\right)+\ln (G)}
$$

Ideally, the gain, $G$, is tuned to $T_{\text {sys }} \times G=1$, so that it exactly matches the undesired losses of the system. The ring-down time, $\tau$, is then only dependent on the losses of the sample. The sensitivity enhancement of the technique was further described by Zhang et al. [32] in a combined experimental and theoretical study. While Zhang et al. and Stewart et al. performed a thorough characterisation of the amplified fibre loop cavity ring-down setup, they did not report on (micro-) analytical measurements. Our report is inspired by these early experiments and we set out to explore the advantages and limitations of using an additional gain element in the fibre loop as a micro-analytical sensor for gases and liquids.

In addition to time-domain measurements, we also tested the possibility of using phase-shift CRD measurements. The phaseshift CRD technique has been described in detail before [33-35] and exploits that intensity-modulated light entering an optical cavity experiences a phase shift (and reduced modulation amplitude) when re-emitted from the cavity. In case of a monoexponential decay process the phase shift is calculated at a given angular modulation frequency, $\Omega$, as

$\phi=-\arctan (\Omega \tau)$

It is important to note that the measurement is most sensitive when $\Omega \tau \approx 1$ and that the phase angle measurement only gives accurate measurements of the ring-down time, when the impulse response is close to a mono-exponential decay [35]. In the present experiments it is therefore important to suppress relaxation oscillations in phase shift measurements.

\section{Experimental setup}

The amplified fibre ring-down setup shown in Fig. 1 uses a two loop system as proposed by Stewart et al. [30]. The two loops share the same erbium doped fibre amplifier (EFDA, KPS-BT-C-18-LN-FA, Keopsys), but operate at two different wavelengths within the EDFA's gain spectrum, which are set by a tuneable bandpass filter (Newport TBF-1550-1.0) and a fibre Bragg grating (FBG 28080940, Advanced Optics Solutions $\mathrm{GmbH}$ ). The relative optical loss is set by a variable optical attenuator (VOA, DD-100, OZ Optics Ltd.) that is placed in the inner loop, whereas the sample and photodetector are placed in the outer loop. In such a setup the gain of the EDFA is "clamped" to the free running lasing threshold of the inner loop, and the outer loop is allowed to "ring up" or "ring down", when driven with a long square light pulse from the tuneable diode laser (TDL, AQ4320D, ANDO). As Stewart et al. previously mentioned a set up based on gain clamping is expected to provide larger stability compared to the simple intracavity laser absorption spectroscopic (ICLAS) measurements [30,36].

In some of the experiments, the inner loop lases at $1534.05 \mathrm{~nm}$ within the gain curve of the EDFA selected using the tuneable BPF. The outer "sample" loop is used for the ring-down detection. The sample loop contains a polarisation controller (PC) to compensate
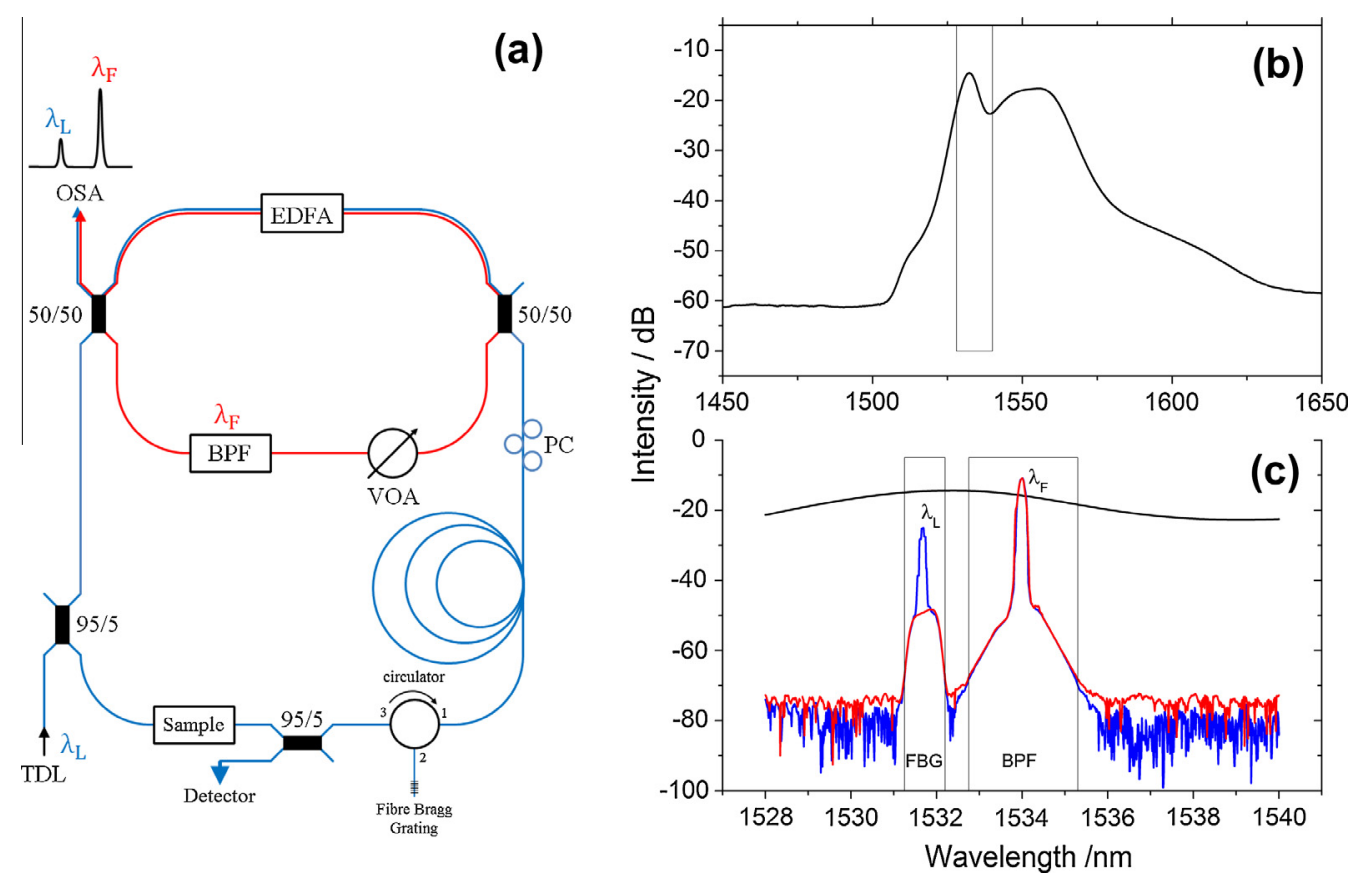

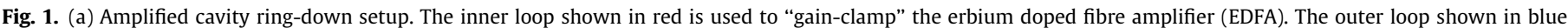

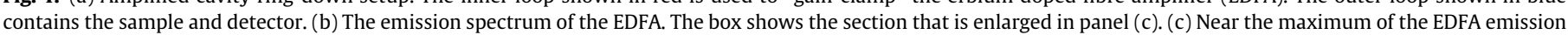

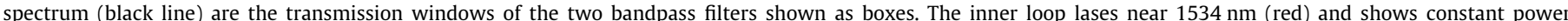

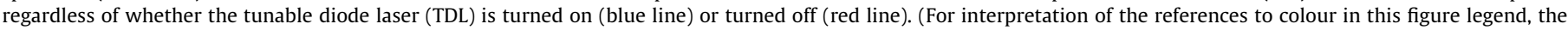
reader is referred to the web version of this article.) 
for birefringence in the fibre. Polarisation changes affect the splitting ratio of the $50 / 50$ couplers that couple light between the inner and outer loop. A spool of $\sim 150 \mathrm{~m}$ fibre optic cable is added to increase the ring-down times by increasing the optical roundtrip path length. The wavelength of the outer loop and thus the wavelength used for the absorbing medium is given as $1532.03 \mathrm{~nm}$ by the fibre Bragg grating (FBG 28080940, Advanced Optics Solutions $\mathrm{GmbH}$ ). The tuneable bandpass filter and fibre Bragg grating were set to different wavelengths to prevent cross talk between the two loops. Light from both loops was coupled to an optical spectrum analyser (OSA, MS9710, Anritsu) so that the relative intensity of the signals could be monitored as well as their respective wavelengths. When the laser was scanned over a larger wavelength region, as described in Section 3.2, a second tunable bandpass filter was used in the sample loop and was adjusted to match the laser wavelength, $\lambda_{L}$.

We used two different sample cells in the outer loop to measure both gaseous and liquid samples. The gas cell was used to detect acetylene and was constructed out of two fibres set $6 \mathrm{~cm}$ apart and terminated by GRIN lenses (Thorlabs) to collimate the light (Fig. 2a). These were surrounded with a gas cell and filled using a gas cylinder containing $1000 \mathrm{ppm}$ of acetylene in helium (PRAXAIR, $1000 \mathrm{ppm}_{2} \mathrm{H}_{2}$ in $\mathrm{He}$, non-certified standard grade). The liquid sensing interface was used to detect 1-octyne (Sigma-Aldrich, 97\%) and 1-dodecyne (Sigma-Aldrich, 98\%) in a non-interacting solvent, such as dodecane (Sigma-Aldrich, $\geqslant 99 \%$ ), and was constructed out of two fibres set $19 \mu \mathrm{m}$ apart with an interrogation volume of $1.0 \mathrm{pL}$ (Fig. 2b).

\section{Results and discussion}

In the following paragraphs we first characterise the experimental setup, by presenting ring-down measurements in the time domain and using phase-shift CRD. Then the "amplified fibre-CRD" technique will be applied to the detection of liquid 1-alkynes through their $\mathrm{C}-\mathrm{H}$ overtone band and to gaseous acetylene through the $v_{1}+v_{3}$ combination band.

\subsection{Characterisation of the amplified FLRDS setup}

Long ring-down times can be obtained using the setup of Fig. 1a. In Fig. 3b we show the ring-down decay trace following a square pulse input from the TDL. The decay can be fit using a biexponential decay function with ring-down times of $\tau_{1}=25.0(2) \mu \mathrm{s}$ and $\tau_{2}=123.4(5) \mu \mathrm{s}$ and relative amplitudes of 34\%:66\%. Considering similar studies on non-amplified fibre-loop CRD spectroscopy, we propose that these decay processes correspond to those occurring in the core and the cladding of the single mode fibre [11]. In the analysis of the loop without the sample cells we characterised the system using the longer ring-down times, i.e. those corresponding to the decay of the core mode. When sample cells were
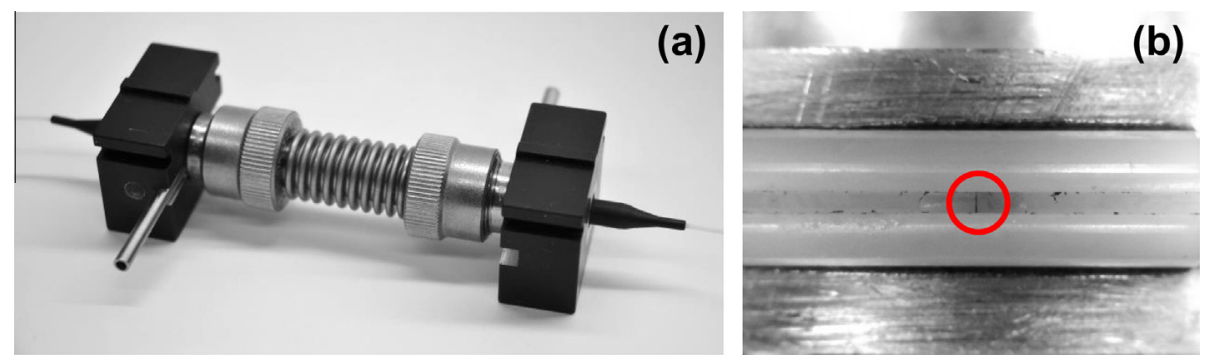

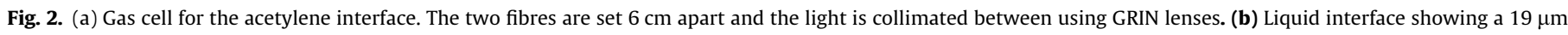
gap (circled) between two single mode fibre ends.

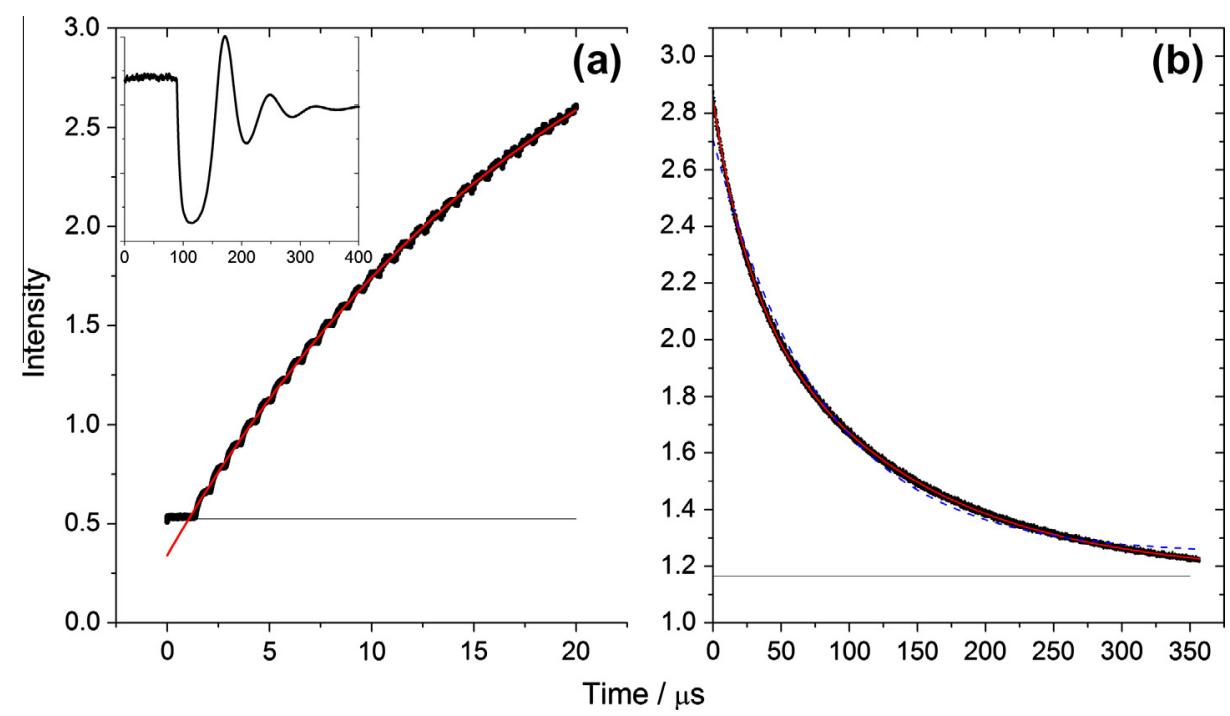

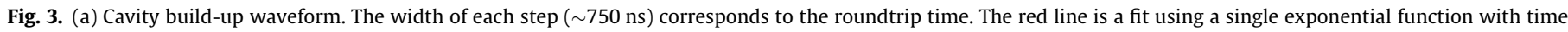

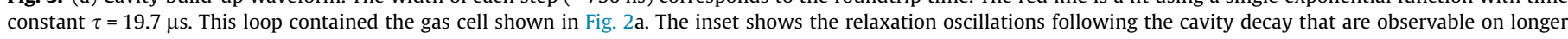

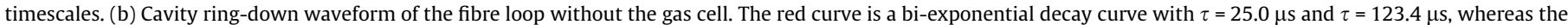

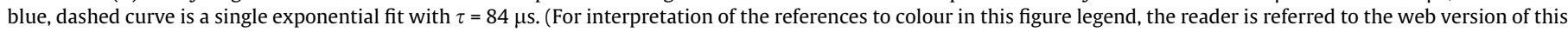
article.) 
inserted in the loop, cladding modes decayed very quickly and their contribution had a much lower amplitude, so that a singleexponential function gave an excellent fit to the cavity ring-up and ring-down curves.

The length of the outer sample loop can be obtained from the steps in the ring-down trace, or - more clearly observable - in the "ring-up" trace of Fig. 3a. The width of each step is about $750 \mathrm{~ns}$, which corresponds to $155 \mathrm{~m}$ length of the outer loop in Fig. $1 \mathrm{a}-$ assuming a refractive index of 1.44 . Fig. 3a gives a ringup time of $19.7 \mu \mathrm{s}$ when the gas cell was inserted. We also recorded the ring-up when the liquid interface was attached as the absorbing sample and found a roundtrip time of 760 ns corresponding to an outer loop length of $158 \mathrm{~m}$ and a ring-down time of $11.5 \mu \mathrm{s}$. As noted by Stewart et al. relaxation oscillations can be observed whenever the probe laser wavelength coincided with the wavelength of the inner loop which is dominated by lasing [31]. Fortunately, as shown in the inset of Fig. 3a, these relaxation oscillations decay much slower compared to the ring-down decay. Therefore, the influence of relaxation oscillations can be reduced by carefully adjusting the relative gain and loss in the two loops using the VOA. Relaxation oscillations can be suppressed by increasing the difference in wavelengths circulating in the two loops. However, since the stability of the ring-down measurement depends on the relative gain of the EDFA in these two wavelength regions, the wavelengths cannot be different by more than a few nanometres. In practice, we set the bandpass filters such that their pass bands do not overlap but are as close as possible. The gain in the outer loop was then increased by adjusting the VOA and by adjusting the amplification of the EDFA, through the pump laser power, such that the relaxation oscillations are visible but fall outside the $200 \mu$ s time window needed to make ring-down measurements.

As expected it was found that when the sample loop was operated at very long ring-down times, and the net loss was close to zero $(T \times G \approx 1)$, the system became very sensitive to mechanical perturbations that might modulate the optical loss. For example, bending the fiber induces not only macrobending losses but also birefringence. The changes of polarisation seem to also change the optical loss in the two loops, possibly because the transmission through the bandpass filters or through fibre-fibre couplers is polarisation sensitive.

\subsection{Ro-vibrational spectroscopy of acetylene gas}

Acetylene was detected previously using an amplified fibre cavity setup using a gas cell similar to that shown in Fig. 2a [37]. This previous work measured the change in ring-down time as the gas partial pressure was increased from $1 \%$ to $20 \%$ at atmospheric pressure. The limit of detection was estimated to be about $0.22 \%$ (2200 ppm) from the measurement error, but this estimate may not have been in accordance to IUPAC recommendations [38]. For the present experiments a tunable bandpass filter was inserted into the sample loop and centred at the maximum of one of the acetylene rotational lines. The laser was then scanned within this pass band. The spectrum of the $\mathrm{P}(13)$ line of the $v_{1}+v_{3}$ combination band is shown in Fig. 4. Here, we performed phase-shift ring-down measurements, i.e. the optical loss is obtained by combining Eqs. (2) and (3) to give

$$
-\ln \left(T_{\text {sample }}\right)=\sigma N d=\frac{\Omega t_{r t}}{\tan \left(\phi-\phi_{0}\right)}+\ln (G)+\ln \left(T_{\text {sys }}\right)
$$

where $N$ is the number density, $d$ is the length of the absorption cell and $\sigma$ is the absorption cross section. It is interesting to note that, without amplification, the "empty cavity" roundtrip loss $-\ln \left(T_{\text {sys }}\right)$ is considerable. From the coupling ratios alone one obtains

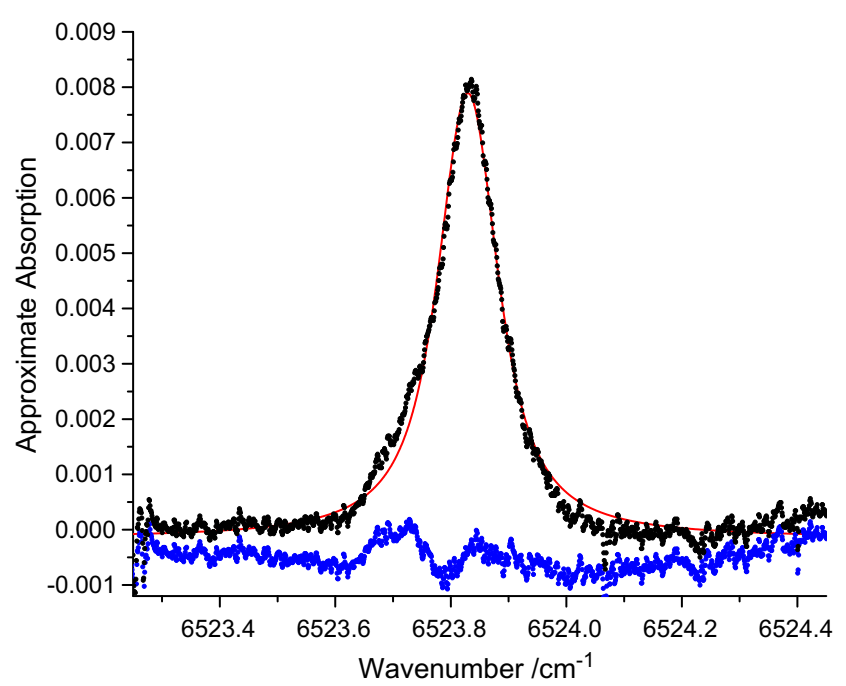

Fig. 4. Phase shift CRD spectrum of the $P(13)$ line of the $v_{1}+v_{3}$ combination band of approximately $1000 \mathrm{ppm}$ acetylene in helium. The original spectrum of $\varphi(\lambda)$ was rescaled using Eq. (4) to be proportional to the absorption, $\sigma N d$. The fit to the Voigt line shape function involves a Lorentzian contribution with a full width at half maximum of $0.11 \mathrm{~cm}^{-1}$ and a Gaussian contribution with a width of $0.046 \mathrm{~cm}^{-1}$. The residual is offset for clarity and shown in blue. (For interpretation of the references to colour in this figure legend, the reader is referred to the web version of this article.)

$T_{\text {sys }}=(0.5)^{2} \times(0.95)^{2}=0.23$. Given an estimated insertion loss of $>50 \%$ for either of the two absorption cells the actual roundtrip loss is probably close to $90 \%$.

Since we have estimates for the number density of $1000 \mathrm{ppm}$ acetylene in helium $\left(N=2.45 \times 10^{16} \mathrm{~cm}^{-3}\right)$ and the absorption length $(d=6.0 \mathrm{~cm})$, we can use Eq. (4) to obtain an order-of-magnitude value for the absorption cross section of a given line. This measurement is complicated by the fact that we cannot easily obtain the contribution to the phase shift of the signal, $\phi_{0}$, that arises from electronic signal delays [34] and we also use square modulated light instead of sine-modulated light which further lowers the accuracy of the phase shift measurement [1]. Nevertheless, one can obtain an estimate for the absorption cross section using time-resolved measurements with $t_{\mathrm{rt}}=750 \mathrm{~ns}$ and the measured off-resonance ring-down time $\tau=50 \mu \mathrm{s}$. These values give $G \times T_{\text {sys }}=0.988$, and together with the known modulation frequency $\Omega=3180 \mathrm{~Hz}$, one obtains a peak absorption cross section of $5.5 \times 10^{-20} \mathrm{~cm}^{2}$ and an integrated absorption cross-section of $1.06 \times 10^{-20} \mathrm{~cm}^{2} \mathrm{~cm}^{-1}$ in fair agreement with the integrated absorption cross section, $0.53 \times 10^{-20} \mathrm{~cm}^{2} \mathrm{~cm}^{-1}$ which was calculated from a Lorentzian fit to the more accurate literature spectrum [39]. While it is, in principle, possible to obtain absolute absorption cross sections using cavity ring-down spectroscopy techniques including amplified CRD spectroscopy - the present setup was not designed to provide calibration-free absorption information.

Of course, it is also possible that the absolute concentration of acetylene was in error, since acetylene is heavier than helium and neither the sample cell nor the acetylene cylinder was agitated before use. Assuming then that the phase shift values were exact, one calculates a number density of $N=4.9 \times 10^{16} \mathrm{~cm}^{-3}$ $\left(2000 \mathrm{ppm}_{\mathrm{v}}\right)$, i.e. a value that seems quite high. It is therefore more likely that the origin of the discrepancy between the previously reported and calculated absorption cross section lies in the inaccuracy of our particular phase shift measurement.

Homogeneous (Doppler) and inhomogeneous (pressure and lifetime) line broadening effects, however, can be quantified using least-square fits to Lorentzian and Voigt line shape functions. Fig. 4 shows that the fit to a Voigt lineshape is dominated by the 
Lorentzian contribution. The FWHM of the Lorentzian contribution $\Delta \tilde{v}_{L}=b P=0.11 \mathrm{~cm}^{-1}$ was used to calculate the pressure broadening coefficient, $b=0.11 \mathrm{~cm}^{-1} / \mathrm{atm}$, which compares satisfactorily with the more accurate value $b=0.0873 \mathrm{~cm}^{-1} /$ atm determined by Arteaga et al. [40]. The less pronounced Gaussian contribution to the Voigt lineshape function is expected to have a FWHM corresponding to

$\Delta \tilde{v}_{G}=\tilde{v} \sqrt{\frac{8 k T \ln 2}{m c_{0}^{2}}}=0.016 \mathrm{~cm}^{-1}$

where $c_{0}$ is the speed of light, $k$ is the Boltzmann constant, $\tilde{v}$ is the central wavelength of the transition, $T$ is the temperature and $m$ is the molecular mass of acetylene. The Gaussian linewidth of $\Delta \tilde{v}_{G}=0.046 \pm 0.004 \mathrm{~cm}^{-1}$ which is obtained from the Voigt fit is considerably larger than the expected value. However, we note that the contribution of Doppler broadening is small and that a Lorentzian fit to the data in Fig. 4 is almost as satisfactory.

Spectra were recorded at a number of other concentrations to determine the limit of detection that may be achieved with the setup. As can be seen from the Lorentzian fit in Fig. 5, acetylene concentrations as low as $\sim 50 \mathrm{ppm}$ can be quantified using the $6 \mathrm{~cm}$ gas cell and even $\sim 25 \mathrm{ppm}$ produces a signal that is $2 \sigma$ above the noise floor.

\subsection{Detection of 1-alkynes in picolitre liquid volumes}

In an attempt to detect liquid samples through weak vibrational overtones in unprecedentedly small detection volumes we opened the fibre-loop and separated the single mode fibre ends by about $19 \mu \mathrm{m}$. The volume between the two fibre end faces is then $230 \mathrm{pL}$, but only a cylinder with a $1.0 \mathrm{pL}$ volume $(8 \mu \mathrm{m}$ diameter and $19 \mu \mathrm{m}$ length) is interrogated by the circulating light. Experiments were conducted using 1-octyne and 1-dodecyne in dodecane at different concentrations. Fig. 6 shows the detection of 1-octyne in the non-absorbing solvent dodecane at $1532.03 \mathrm{~nm}$. Five different concentrations of 1-octyne in dodecane ranging from $100 \%$ to $10 \%$ were sampled randomly to demonstrate the repeatability of the measurement and to check for slow drifts and cross talk between samples. The solid red lines correspond to the $3 \sigma$ level of the background signal and it is apparent that

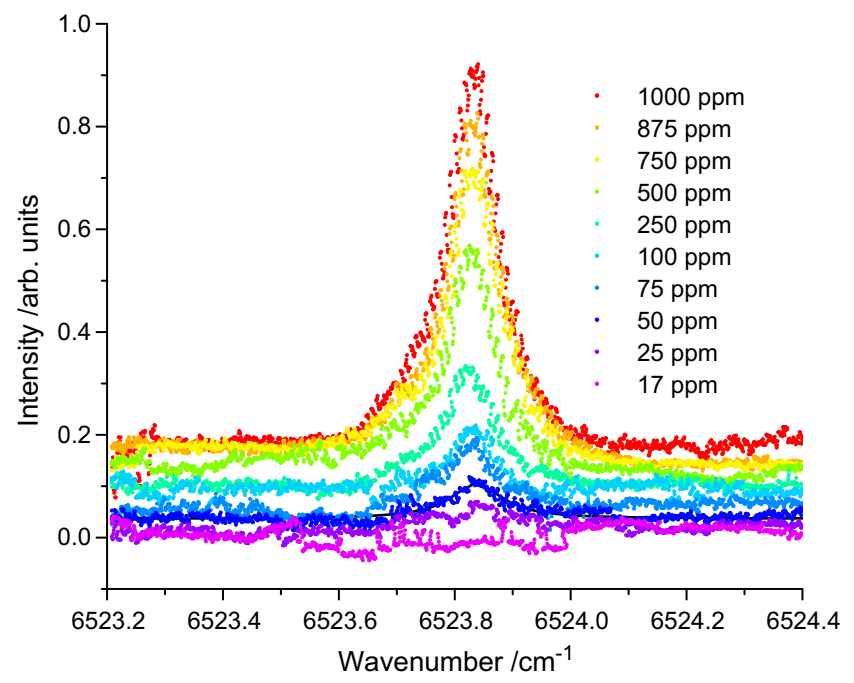

Fig. 5. Spectrum of the $P(13)$ line as in Fig. 4 but recorded at different partial pressures of acetylene in helium. The solid black line is a fit of the absorption line at $\sim 50 \mathrm{ppm}$ to a Lorentzian line shape function. The curves are shifted vertically for clarity (reproduced from Ref. [1] according to the Creative Commons Attribution License).

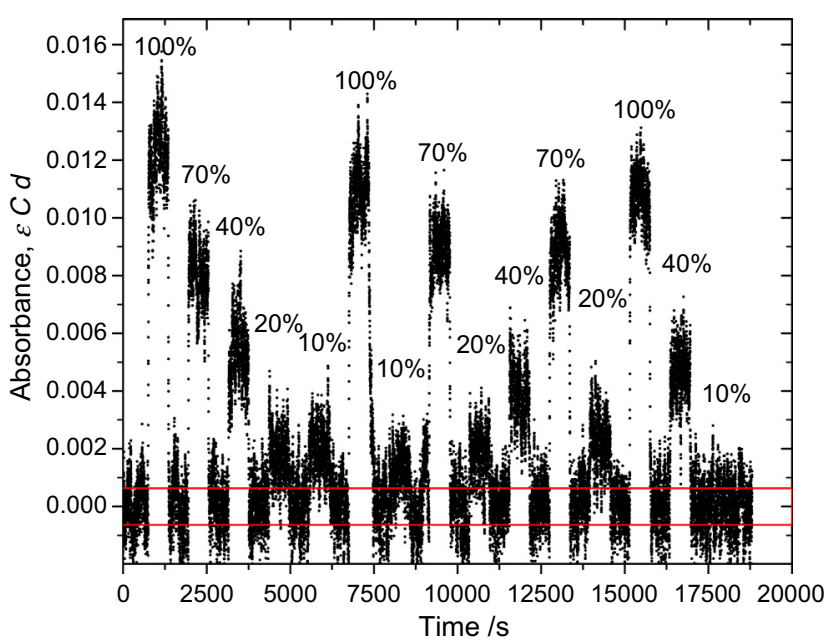

Fig. 6. 1 -Octyne in dodecane at concentrations $100 \%, 70 \%, 40 \%, 20 \%$ and $10 \%$ was injected into the interface of Fig. $2 \mathrm{~b}$. The solid red lines signify the background noise at the $3 \sigma$ level. (For interpretation of the references to colour in this figure legend, the reader is referred to the web version of this article.)

the detection limit is better than $20 \%$ of 1 -octyne in dodecane. Similar responses are obtained for 12 injections of 1-dodecyne in dodecane at 4 different concentrations (not shown). Fig. 7 shows linear relationships between the measured absorption loss and the concentration for both absorbers. In Figs. 6 and 7 the absorption of the sample is calculated from Eq. (2) and $-\ln \left(T_{\text {sample }}\right)=\varepsilon C d$ using

$-\ln \left(T_{\text {sample }}\right)=\frac{t_{r t}}{\tau}-\frac{t_{r t}}{\tau_{0}}$

where $C$ is the concentration. The pathlength through the sample, $d \approx 19 \mu \mathrm{m}$, was determined from the absorption cross section of $1.6 \times 10^{-19} \mathrm{~cm}^{-2}$ of the $\mathrm{C}-\mathrm{H}$ stretch overtone located at around $1532 \mathrm{~nm}$ [12]. The roundtrip time, $t_{\mathrm{rt}}=760 \mathrm{~ns}$ was calculated from the steps in the ring-up curve (akin to Fig. 3a) and the ring-down time of the system without absorber, $\tau_{0}$, was determined from the baseline

$$
\tau_{0}=-\frac{t_{r t}}{\ln (G)+\ln \left(T_{s y s}\right)}
$$

The limit of detection can also be determined from the calibration curves (Fig. 7). The error bars $(1 \sigma)$ shown in Fig. 7 were calculated from three average signal levels obtained from the injection at each of the 5 or 4 concentrations, whereas the $3 \sigma$ confidence intervals for the linear fit were obtained as described previously [38]. The limit of detection in this case is calculated using the minimal detectable absorbance and corresponds to $15-20 \%$ of alkyne in dodecane in both cases and is in good agreement with the estimate of Fig. 6. We note that the minimal detectable absorptivity of $<10^{-4}$ is comparable with the lowest values obtained to date for micro-analytical liquid absorption spectroscopy [19]. The minimal detectable absorptivity, $(\varepsilon C)_{\min }=0.033 \mathrm{~cm}^{-1}$ is comparable to that of many devices based on either fibre loops or liquid core waveguides, whereas the minimal detectable absorption cross section $\mathrm{V}_{\mathrm{det}}(\varepsilon C)_{\min }=0.075 \mu \mathrm{m}^{2}$, is to the best of our knowledge lower than that of any previously reported absorption technique by more than one order of magnitude.

\section{Conclusion}

Amplified fibre-loop cavity ring-down (CRD) was used to determine detection limits of both gaseous species and liquid samples. Similar to earlier work we used two nested fibre loops that shared 


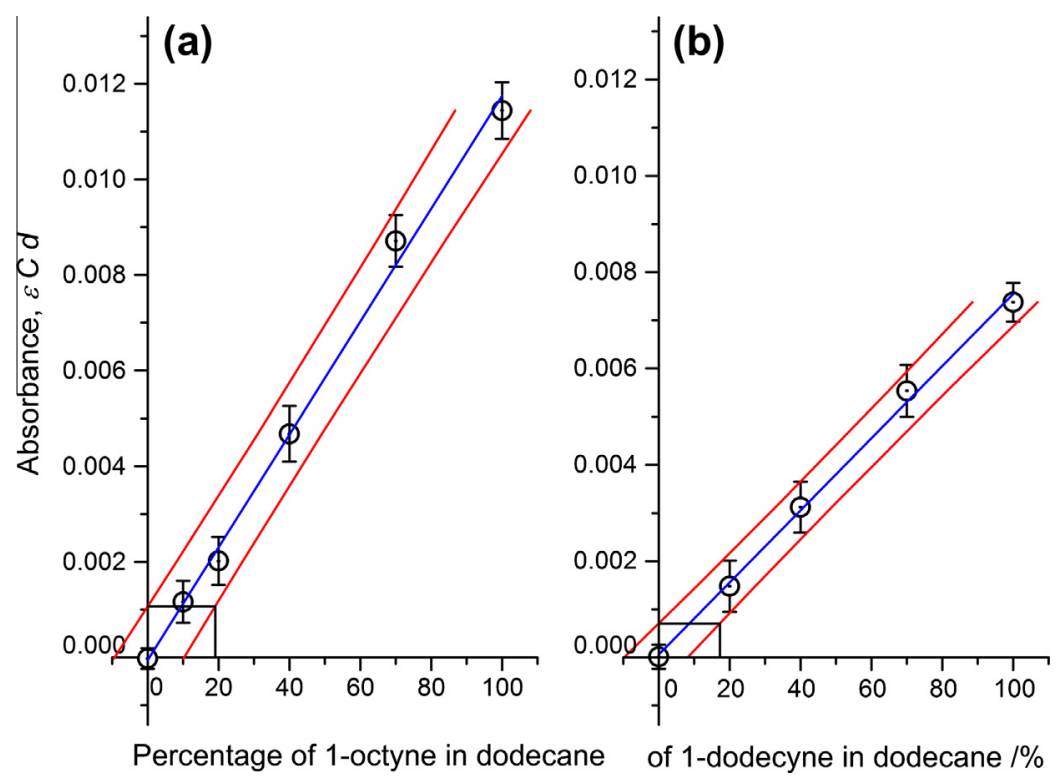

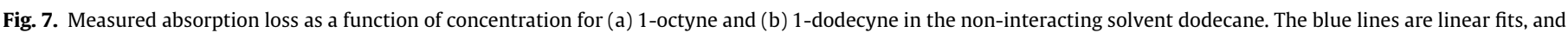

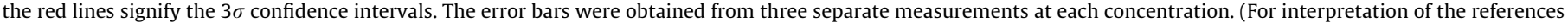
to colour in this figure legend, the reader is referred to the web version of this article.)

a fibre amplifier. The system was built from commercial off-theshelf equipment and equipped with a home-built sample introduction system. Despite the weakness of the combination band of the acetylene sample and the overtone band of the 1-alkyne samples, amplified CRD spectroscopy was able to quantify gaseous acetylene in helium at concentrations of $50 \mathrm{ppm}$ using the $\mathrm{P}(13)$ branch and permitted rotational lineshape analysis. Liquid samples of 1octyne and 1-dodecyne were detected at a limit of detection corresponding to $20 \%$ in the non-absorbing solvent dodecane. While the minimal detectable concentration is 20 -fold higher than what had been achieved before [12], we note that our detection volume is close to 1.0 picolitres and is orders of magnitude smaller than that reported in previous work. Despite being somewhat sensitive to polarisation changes and mechanical perturbations, amplified cavity ring-down spectroscopy shows resilience to light intensity changes and a greatly enhanced absorption path using cavities that may exhibit very high (passive) roundtrip loss.

\section{Acknowledgments}

This work was financially supported by the Natural Sciences and Engineering Research Council (NSERC) of Canada. The authors thank Stephen Walker for his help in interpreting the acetylene lineshapes and absorption cross sections.

\section{References}

[1] H. Waechter, J. Litman, A.H. Cheung, J.A. Barnes, H.-P. Loock, Sensors 10 (2010) 1716-1742.

[2] A. O'Keefe, D. Deacon, Rev. Sci. Instrum. 59 (1988) 2544.

[3] P. Zalicki, R.N. Zare, J. Chem. Phys. 102 (1995) 2708-2717.

[4] K.E. Whittaker, L. Ciaffoni, G. Hancock, R. Peverall, G.A.D. Ritchie, Appl. Phys. B 109 (2012) 333-343.

[5] T.J.A. Butler, D. Mellon, J. Kim, J. Litman, A.J. Orr-Ewing, J. Phys. Chem. A 113 (2009) 3963-3972.

[6] T.J.A. Butler, J.L. Miller, A.J. Orr-Ewing, J. Chem. Phys. (2007) 126.

[7] G. Berden, R. Peeters, G. Meijer, Int. Rev. Phys. Chem. 19 (2000) 565-607.

[8] S. Xu, G. Sha, J. Xie, Rev. Sci. Instrum. 73 (2002) 255.

[9] L. van der Sneppen, F. Ariese, C. Gooijer, W. Ubachs, Ann. Rev. Anal. Chem. 2 (2009) 13-35.

[10] R.S. Brown, I. Kozin, Z. Tong, R.D. Oleschuk, H.-P. Loock, J. Chem. Phys. 117 (2002) 10444-10447.
[11] Z.G. Tong, M. Jakubinek, A. Wright, A. Gillies, H.-P. Loock, Rev. Sci. Instrum. 74 (2003) 4818-4826

[12] P.B. Tarsa, P. Rabinowitz, K.K. Lehmann, Chem. Phys. Lett. 383 (2004) 297-303.

[13] R.K. Li, H.-P. Loock, R.D. Oleschuk, Anal. Chem. 78 (2006) 5685-5692.

[14] H.-P. Loock, TrAC, Trends Anal. Chem. 25 (2006) 655-664.

[15] J.A. Barnes, M. Dreher, K. Plett, R.S. Brown, C.M. Crudden, H.-P. Loock, Analyst 133 (2008) 1541.

[16] H. Waechter, K. Bescherer, C. Dürr, R.D. Oleschuk, H.-P. Loock, Anal. Chem. 81 (2009) 9048.

[17] C.M. Rushworth, D. James, J.W.L. Lee, C. Vallance, Anal. Chem. 83 (2011) 84928500.

[18] D. James, B. Oag, C.M. Rushworth, J.W.L. Lee, J. Davies, J.T. Cabral, C. Vallance, RSC Advances 2 (2012) 5376-5384.

[19] K. Bescherer, J.A. Barnes, H.-P. Loock, Anal. Chem. 85 (2013) 4328-4334.

[20] K.L. Snyder, R.N. Zare, Anal. Chem. 75 (2003) 3086-3091.

[21] K.L. Bechtel, R.N. Zare, A.A. Kachanov, S.S. Sanders, B.A. Paldus, Anal. Chem. 77 (2005) 1177-1182

[22] B. Bahnev, L. van der Sneppen, A.E. Wiskerke, F. Ariese, C. Gooijer, W. Ubachs, Anal. Chem. 77 (2005) 1188-1191.

[23] L. van der Sneppen, A. Wiskerke, F. Ariese, C. Gooijer, W. Ubachs, Anal. Chim. Acta 558 (2006) 2-6.

[24] C.M. Rushworth, J. Davies, J.T. Cabral, P.R. Dolan, J.M. Smith, C. Vallance, Chem. Phys. Lett. 554 (2012) 1-14.

[25] H.-P. Loock, in: G. Berden, R. Engeln (Eds.), Cavity Ring-Down Spectroscopy: Techniques and Applications, Blackwell Publishing, 2009, pp. 113-144.

[26] P.B. Tarsa, A.D. Wist, P. Rabinowitz, K.K. Lehmann, Appl. Phys. Lett. 85 (2004) 4523-4525.

[27] M. Gupta, H. Jiao, A. O’Keefe, Opt. Lett. 27 (2002) 1878-1880.

[28] M. Andachi, T. Nakayama, M. Kawasaki, S. Kurokawa, H.-P. Loock, Appl. Phys. B 88 (2007) 131-135.

[29] H. Waechter, D. Munzke, A. Jang, H.-P. Loock, Anal. Chem. 83 (2011) 27192725.

[30] G. Stewart, K. Atherton, H.B. Yu, B. Culshaw, Meas. Sci. Technol. 12 (2001) 843849.

[31] G. Stewart, K. Atherton, B. Culshaw, Opt. Lett. 29 (2004) 442-444.

[32] Y. Zhang, Z. Min, J. Wei, Sens. Act., B: Chem. 104 (2003) 183-187.

[33] R. Engeln, G. von Helden, G. Berden, G. Meijer, Chem. Phys. Lett. 262 (1996) 105-109.

[34] Z. Tong, A. Wright, T. McCormick, R. Li, R.D. Oleschuk, H.-P. Loock, Anal. Chem. 76 (2004) 6594-6599.

[35] K. Bescherer, J.A. Barnes, S. Dias, G. Gagliardi, H.-P. Loock, N.R. Trefiak, H. Waechter, S. Yam, Appl. Phys. B 96 (2009) 193-200.

[36] G. Stewart, P. Shields, B. Culshaw, Meas. Sci. Technol. 15 (2004) 1621-1628.

[37] Y. Zhang, M. Zhang, W. Jin, H.L. Ho, M.S. Demokan, B. Culshaw, G. Stewart, Opt. Commun. 232 (2004) 295-301.

[38] H.-P. Loock, P.D. Wentzell, Sens. Act., B: Chem. 173 (2012) 157-163.

[39] S.W. Sharpe, T.J. Johnson, R.L. Sams, P.M. Chu, G.C. Rhoderick, P.A. Johnson, Appl. Spectrosc. 58 (2004) 1452-1461.

[40] S.W. Arteaga, C.M. Bejger, J.L. Gerecke, J.L. Hardwick, Z.T. Martin, J. Mayo, E.A. Mcllhattan, J.M.F. Moreau, M.J. Pilkenton, M.J. Polston, B.T. Robertson, E.N. Wolf, J. Mol. Spectrosc. 243 (2007) 253-266. 